\title{
Eurasian cooling in response to Arctic sea-ice loss is not proved by maximum covariance analysis
}

Article

Accepted Version

Zappa, G., Ceppi, P. and Shepherd, T. G. (2021) Eurasian cooling in response to Arctic sea-ice loss is not proved by maximum covariance analysis. Nature Climate Change, 11. pp. 106-108. ISSN 1758-678X doi: https://doi.org/10.1038/s41558020-00982-8 Available at https://centaur.reading.ac.uk/95136/

It is advisable to refer to the publisher's version if you intend to cite from the work. See Guidance on citing.

To link to this article DOI: http://dx.doi.org/10.1038/s41558-020-00982-8

Publisher: Nature Publishing Group

All outputs in CentAUR are protected by Intellectual Property Rights law, including copyright law. Copyright and IPR is retained by the creators or other copyright holders. Terms and conditions for use of this material are defined in the End User Agreement.

$\underline{\text { www.reading.ac.uk/centaur }}$ 
Central Archive at the University of Reading

Reading's research outputs online 


\title{
Eurasian cooling in response to Arctic sea-ice loss is not proved by maximum covariance analysis
}

\author{
Giuseppe Zappa ${ }^{1,2}$, Paulo Ceppi ${ }^{3}$, and Theodore G. Shepherd ${ }^{1}$ \\ ${ }^{1}$ Department of Meteorology, University of Reading, Reading RG6 6BB, UK \\ ${ }^{2}$ Istituto di Scienze dell'Atmosfera e del Clima, Consiglio Nazionale delle Ricerche (ISAC-CNR), \\ Bologna 40129, Italy \\ ${ }^{3}$ Grantham Institute for Climate Change and the Environment, Imperial College, London SW7 $2 A Z$, \\ $U K$
}

The extent to which the ongoing decline in Arctic sea ice affects mid-latitude climate has received great attention and polarised opinions. The basic issue is whether the inter-annual variability in Arctic sea ice is the cause of, or the response to, variability in mid-latitude atmospheric circulation [1]. Mori et al. (M19, [2]) claims to have reconciled previous conflicting studies by showing that a consistent mid-latitude climate response to inter-annual sea-ice anomalies can be identified between the ERA-Interim reanalysis, taken as observations, and an ensemble of atmosphere-only (AMIP) climate model simulations. Here we demonstrate that such a conclusion cannot be drawn, due to issues with the interpretation of the maximum covariance analysis performed. After applying the M19 approach to the output from a simple statistical model, we conclude that a predominant atmospheric forcing of the sea-ice variability, rather than the converse, is a more plausible explanation of the results presented in M19.

A leading mode of internal atmospheric variability is associated, in its positive phase, with a Siberian anticyclone, a Warm Arctic and Cold Eurasia (WACE mode, Fig 1d in M19). It is debated whether anomalies in the extent of Barents and Kara sea ice can modulate the frequency of occurrence of this mode, given that the Siberian circulation anomaly could itself force sea-ice anomalies by warming the Arctic region. To discriminate between these two possible scenarios, M19 rely on identifying a mode of year-to-year co-variability in the winter-mean (DJF) Eurasian surface temperature between the ERA-Interim reanalysis and an ensemble of AMIP simulations, i.e. climate runs forced by observed oceanic conditions (including sea ice). The approach is well designed: if sea ice forces circulation, and models are realistic, the WACE modes in the ERAInterim and AMIP simulations should covary in time. If instead sea ice merely responds to circulation, the WACE modes should not covary: in the real world the WACE mode would force sea-ice variability, while in the AMIP simulations the imposed observed sea ice would only force a monopole of Arctic temperature variability via local thermodynamic processes.

The leading mode of Eurasian surface temperature co-variability between ERA-Interim and the AMIP simulations is identified in M19 via maximum covariance analysis (MCA), as implemented through the singular value decomposition (SVD) of the covariance matrix between the two surface temperature fields in 0E-180E, 20N-90N. However, the pair of singular vectors that comprise the co-varying mode are not displayed in the paper. Instead, the authors discuss 
the mode in terms of the homogeneous regression maps obtained by regressing each field on the expansion coefficient (EC) of its own singular vector (see Supplementary Information for an overview of the methodology). The homogeneous regression maps are not necessarily directly related to the singular vectors, and hence to the structure of the co-varying mode $[3,4]$. This is because the ECs are obtained by projecting the two analysed fields on their own singular vectors. Hence, in addition to reflecting the co-varying mode, the ECs include variance generated by any internal mode of variability that is not orthogonal to the singular vectors themselves. When the original fields are regressed on their own ECs, such internal modes can be aliased into the homogeneous regression maps. The way to isolate the structure of the co-varying mode is via heterogeneous regression maps, in which each field is regressed on the EC from the other field $[3]$.

The potential pitfalls of solely examining homogeneous maps are explored by applying the statistical method from M19 to the output from a simple statistical model that qualitatively incorporates the influence of sea ice and of the WACE mode on surface temperature variability. In the simple model, the direction of the interaction between atmospheric circulation and sea ice can be directly controlled (see SI). Regardless of whether sea-ice variability forces atmospheric circulation or vice-versa, we find that the homogeneous regressions for the leading co-varying mode always show WACE-like patterns characterised by a warm Arctic, a cold Eurasia and a positive Siberian surface pressure anomaly. Because they alias in the internal variability in the WACE mode, homogeneous regressions are insufficient to discriminate between these different scenarios from the simple model. The same is not true for the heterogeneous regressions, which correctly identify distinct pairs of co-varying patterns - either WACE-like or an Arctic Temperature Monopole (ATM) - depending on the presence and direction of the interaction between sea ice and the atmospheric circulation (Table 1).

We therefore compare the structure of the co-varying mode between the ERA-Interim and AMIP simulations obtained from the homogeneous (Fig. 1a-b) and heterogeneous (Fig. 1c-f) regression maps (see also the singular vectors in Extended Data Fig. 1). The only difference with M19 is that, since the MIROC4 simulations are unavailable to the authors, the AMIP multimodel ensemble consists of 6 rather than 7 models. Nonetheless, the homogeneous maps bear a strong resemblance to those presented in M19, featuring the WACE-mode temperature dipole between the Arctic and Central Eurasia together with the Siberian sea level pressure anomalies. All these three features are present, albeit with a weaker amplitude, in the heterogeneous map from ERA-Interim (Fig. 1c,e), but not in the heterogeneous map from the AMIP ensemble (Fig. 1d,f). In particular, while the warm anomaly in the Barents and Kara seas is still present due to thermodynamic forcing from sea ice, the cold anomaly in Central Eurasia is weakened and displaced southward and, most importantly, the Siberian anticyclonic anomaly is entirely missing (Fig. 1f). This implies the co-varying temperature pattern in the AMIP simulations is distinct from the WACE mode, since the weak cold anomalies that persist further south in Eurasia are not generated via cold advection by the Siberian anticyclone anomaly.

The missing Siberian SLP signature of the WACE mode in the heterogeneous maps is found in all individual models, with the possible exception of ECHAM5, where the signal is nonetheless not significant (Extended Data Fig. 2). In contrast, the SLP signal is present in all models' homogeneous maps, which we attribute to the aliasing of internal variability that affects Arctic temperature. Indeed, the magnitude of both the Siberian circulation and Eurasian temperature 
signals in the homogeneous maps are reduced, and made more similar to the heterogeneous maps, by averaging the models' ensemble members before the MCA (Supplementary Fig. 1). This would not be expected if the signal was forced, since ensemble averaging only suppresses the unforced internal variability.

The exact cause of the remaining southward-displaced cold anomaly in southern Eurasia is unknown, but we note that the co-varying mode is associated with a global pattern of SST anomalies (Extended Data Fig. 3). Some of these SST anomalies could drive southern Eurasian temperature and circulation co-variability without the need to invoke an Arctic mechanism (see Supplementary Fig 2 on the role of tropical Pacific SSTs). Understanding and isolating these connections should be a topic for future research.

Comparing the structure of the heterogeneous maps between the ERA-Interim and the AMIP simulations with those from the statistical model, suggests that a more plausible interpretation of the results in M19 is an atmospheric driving - via the WACE mode - of Barents and Kara sea-ice variability in the real world (Ice $\leftarrow$ Atm). This interpretation is consistent with the comment by Screen and Blackport, 2019 [5], who examined the lead-lag covariance between sea ice and the WACE mode. M19 argue for the opposite direction of causality based on the negative correlation $(r)$ in the year-to-year variability between the sea ice and the ECs, and later use $r^{2}$ to quantify the fraction of WACE variance that is forced from sea ice. However, the rationale is flawed since, as shown by the statistical model, the correlation between the ECs and the sea-ice anomalies is always negative regardless of whether sea ice forces circulation, or vice-versa (Table 1). This is a direct consequence of the fact that sea-ice anomalies directly affect Arctic temperature, hence projecting on the singular vector.

Another potential interpretation of Fig. 1 is that the models entirely fail to capture the observed dynamical WACE response to sea-ice anomalies. But in that case, the models certainly cannot be used to argue in favour of a causal linkage. Either way, the conclusion of M19 is not supported by the evidence presented.

Acknowledgments: The authors acknowledge Masato Mori and Hisashi Nakamura for useful feedback, and two reviewers for their insightful comments. GZ and TGS were supported by the ERC advanced grant 339390. PC was supported by an Imperial College Research Fellowship and NERC grant NE/T006250/1.

Data availability: The ERA-Interim reanalysis is publicly available from ECMWF (https:// www.ecmwf.int/en/forecasts/datasets/reanalysis-datasets/era-interim). The AMIP FACTS simulations are publicly available from NOAA (https://www.esrl.noaa.gov/psd/ repository/alias/factsdocs).

Author contributions: GZ conceived the study and performed the analyses. All authors contributed to interpreting the results and writing the manuscript.

Competing interests: The authors declare no competing interests.

Corresponding author: Correspondence and requests for materials should be addressed to GZ. 


\begin{tabular}{|c|c|c|c|c|c|c|}
\hline & \multicolumn{2}{|c|}{ Ice $\rightarrow$ Atm } & \multicolumn{2}{|c|}{ Ice $\nsim$ Atm } & \multicolumn{2}{|c|}{ Ice $\leftarrow$ Atm } \\
\hline & Real & AMIP & Real & AMIP & Real & AMIP \\
\hline & \multicolumn{6}{|c|}{ Homogeneous regression } \\
\hline$T_{\text {Arctic }}$ & 2.2 & 2.2 & 1.4 & 1.4 & 2.2 & 1.7 \\
\hline$C$ & 1.4 & 1.4 & 0.7 & 0.7 & 0.9 & 0.6 \\
\hline$T_{\text {Asia }}$ & -1.4 & -1.4 & -0.7 & -0.7 & -0.9 & -0.6 \\
\hline \multirow[t]{2}{*}{ Pattern } & WACE & WACE & WACE & WACE & WACE & WACE \\
\hline & \multicolumn{6}{|c|}{ Heterogeneous regression } \\
\hline$T_{\text {Arctic }}$ & 2.3 & 2.3 & 1.4 & 1.4 & 2.3 & 1.7 \\
\hline$C$ & 1.2 & 1.2 & 0.0 & 0.0 & 0.8 & 0.0 \\
\hline$T_{\text {Asia }}$ & -1.2 & -1.2 & 0.0 & 0.0 & -0.8 & 0.0 \\
\hline \multirow[t]{2}{*}{ Pattern } & WACE & WACE & ATM & ATM & WACE & ATM \\
\hline & \multicolumn{6}{|c|}{ Correlation between sea ice (I) and ECs } \\
\hline$r_{I, E C}$ & -0.86 & -0.86 & -0.71 & -0.71 & -0.93 & -0.82 \\
\hline
\end{tabular}

Table 1: The application of the M19 approach to the output from a simple statistical model. The modes of co-variability between the "real-world" (Real) and "AMIP-world" (AMIP) systems are described using homogeneous (top) and heterogeneous (bottom) regressions in terms of the following variables from the simple model (see Supplementary Information for details): $T_{\text {Arctic }}$ (representing Arctic temperature), $C$ (atmospheric circulation, positive for a Siberian anticyclone) and $T_{\text {Asia }}$ (Central Eurasian temperature). The patterns are classified either as WACE-like (WACE), if formed by a temperature dipole and a circulation anomaly, or as an Arctic Temperature Monopole (ATM) with no circulation anomaly (also highlighted bold). Note that the exact values are of secondary importance. The columns report results from different setups of the statistical model: sea ice driving the circulation (Ice $\rightarrow$ Atm), no interaction between sea ice and circulation (Ice $\nsim$ Atm), and circulation driving the sea ice (Ice $\leftarrow$ Atm). The bottom line reports the correlation between the variability in the sea ice (I) and that in the expansion coefficients. Only the heterogeneous maps can discriminate between the different model setups: if the WACE pattern is found in both the real-world and the AMIPworld, then sea ice forces circulation, while if the WACE pattern is found in the real-world only, then it is the atmosphere that forces the sea ice. 
a) ERAI - homogeneous map (TAS)

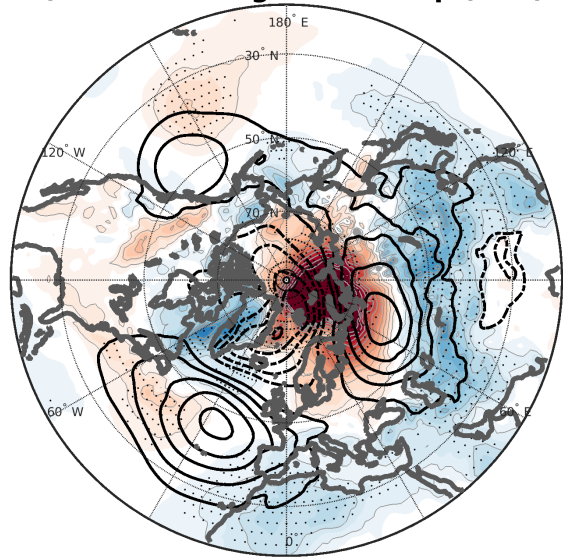

c) ERAI - heterogeneous map (TAS)

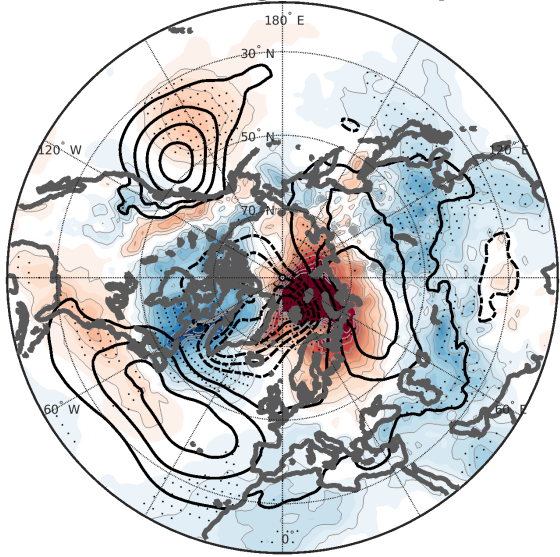

e) ERAI - heterogeneous map (SLP)

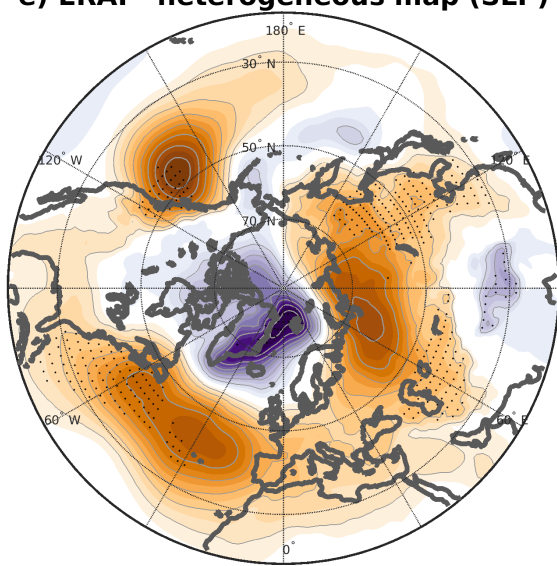

b) AMIP - homogeneous map (TAS)

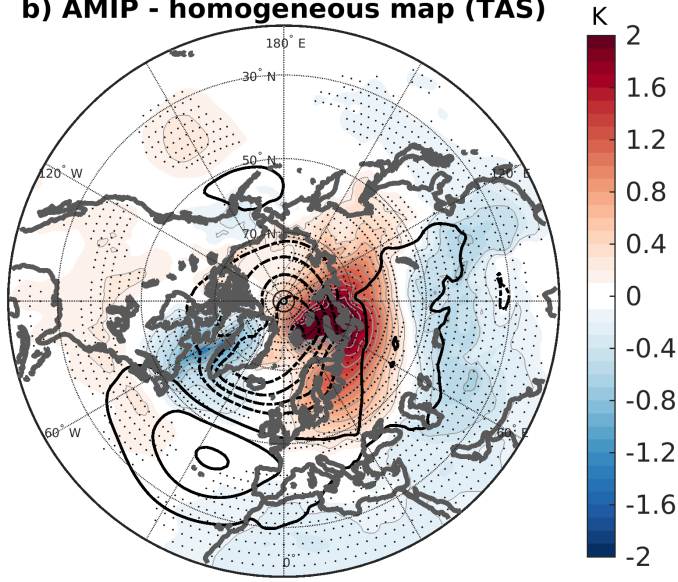

d) AMIP - heterogeneous map (TAS)

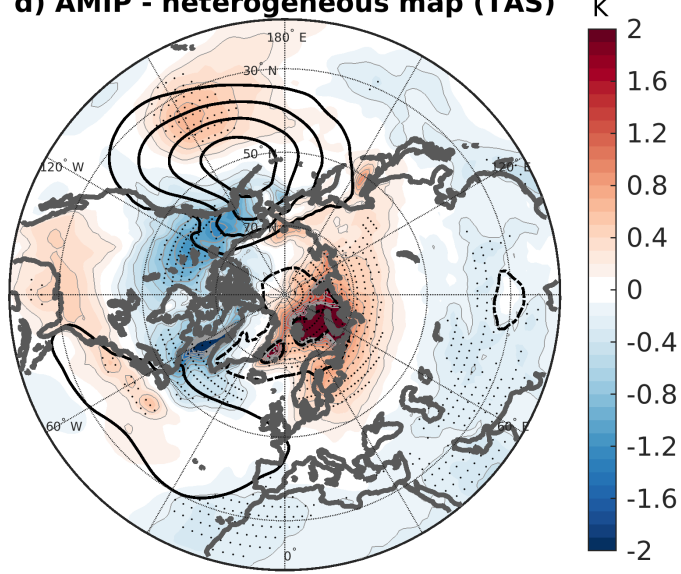

f) AMIP - heterogeneous map (SLP)

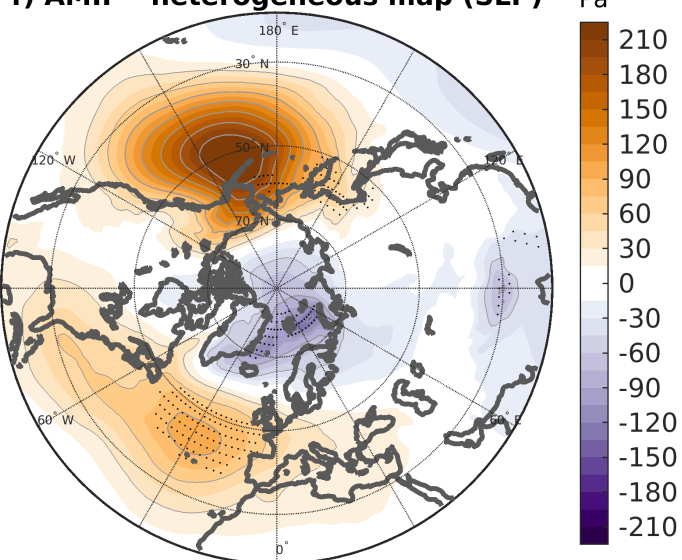

Figure 1: The co-varying mode of Eurasian surface temperature between ERAInterim and the AMIP simulations. Comparison between $a-b)$ the homogeneous and $c-f$ ) heterogeneous regression maps following the analysis in M19. a,c,e) refer to the ERA-Interim reanalysis, while b,d,f) refer to the AMIP simulations. In a-d), shading shows the near-surface atmospheric temperature $(\mathrm{K})$, and the contours the sea-level pressure with a c.i. of $0.5 \mathrm{hPa}$, solid for positive and dashed for negative. The heterogeneous map for sea-level pressure is further shown in e-f) in shading. Stippling indicates statistical significance at the $5 \%$ level in the shaded variable, i.e. temperature in a-d) and pressure in e-f), as obtained by bootstrapping the individual years with replacement (see SI). All maps are scaled to one standard deviation anomaly in the expansion coefficients. Note how only the heterogeneous maps correctly reproduce the co-varying temperature signals shown by the singular vectors within their domain of definition in the Eastern Hemisphere (Extended Data Fig. 1). 
a) ERAI - singular vector

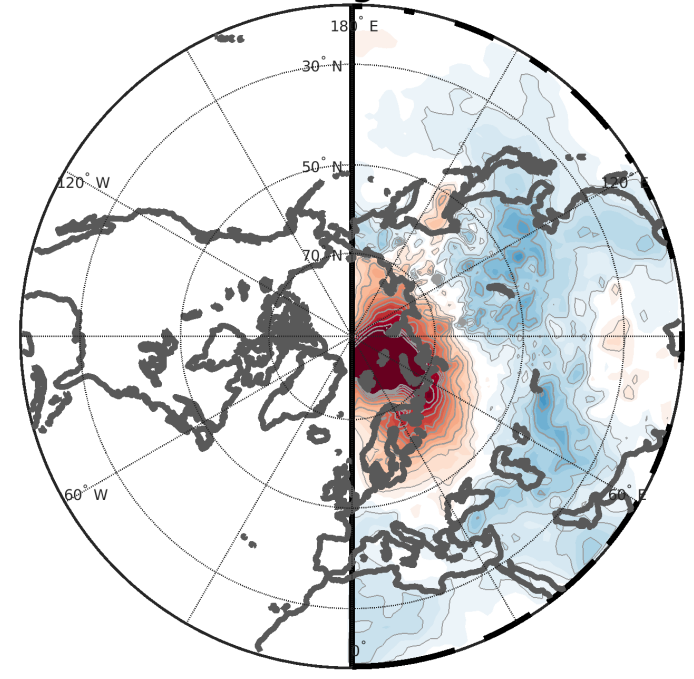

b) AMIP - singular vector

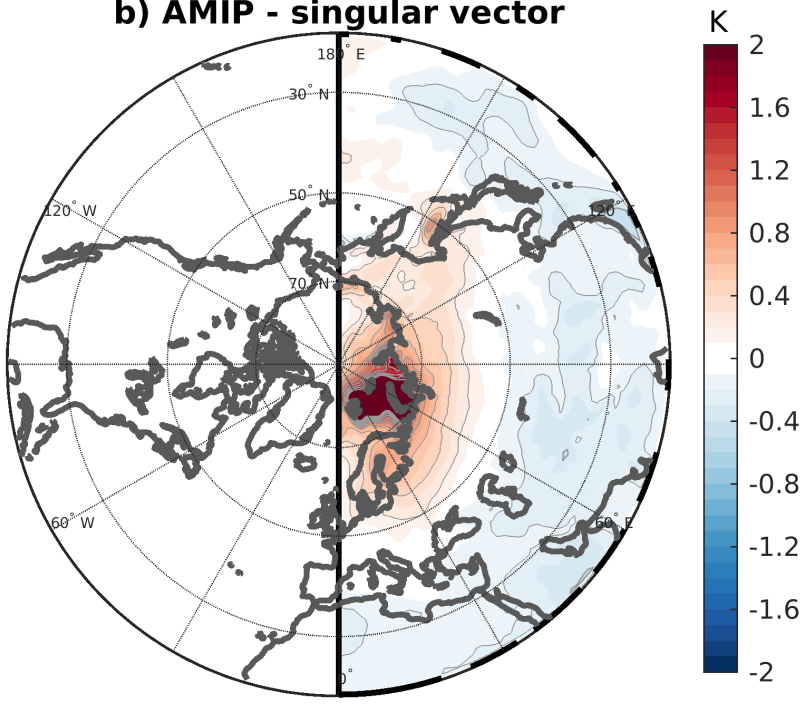

Extended Data Figure 1: The singular vectors. The pair of singular vectors composing the first co-varying surface temperature mode between the a) ERA-Interim and b) AMIP simulations via the maximum covariance analysis proposed in M19. As in Fig. 1, the vectors are scaled to correspond to unit standard deviation in the expansion coefficients. 


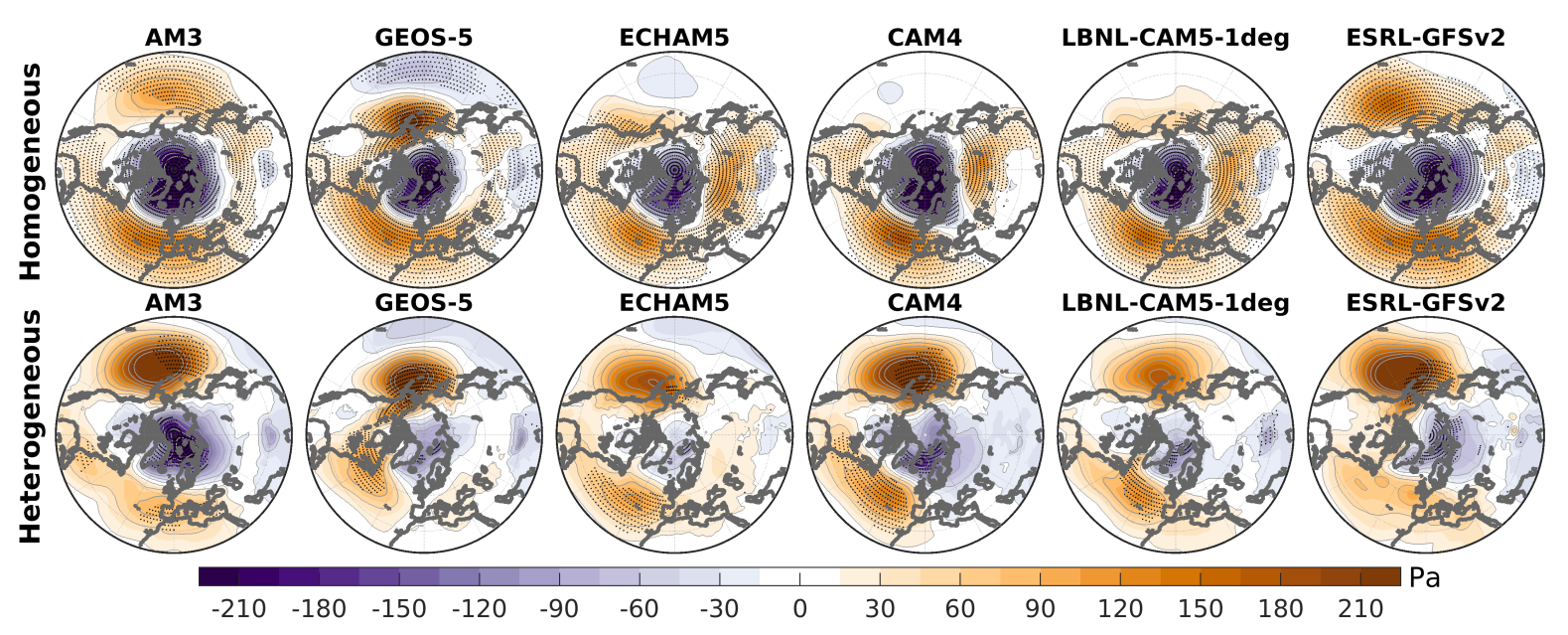

Extended Data Figure 2: The robustness of the co-varying mode to model differences. Homogeneous (top) and heterogeneous (bottom) regression maps of sea level pressure in the AMIP simulations obtained by separately performing the maximum covariance analysis for each individual model and using all the available ensemble members: 17 members are used for AM3, 12 for GEOS-5, 20 for CAM4, and 50 for all other models. Stippling shows statistical significance at the $5 \%$ level as in Fig. 1. 


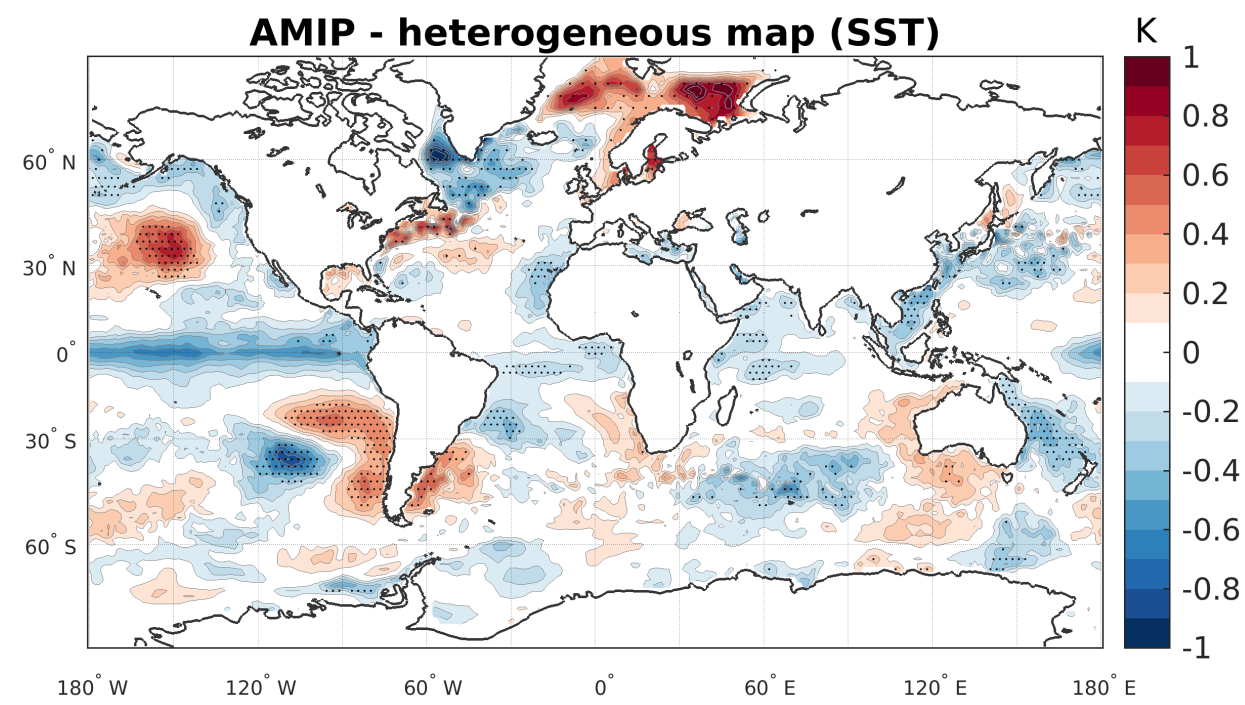

Extended Data Figure 3: The potential confounding role of the SSTs. Heterogeneous map of the SSTs associated to the co-varying mode in the AMIP simulations. Stippling denotes statistical significance at the $5 \%$ level. The potential of these SST anomalies, such as the ENSOlike pattern in the tropical Pacific, to force the circulation signals associated to the co-varying mode in Fig. 1f is discussed in the SI (Supplementary Fig. 2). 


\section{References}

[1] T. G. Shepherd, "Effects of a warming Arctic," Science, vol. 353, pp. 989-990, 2016.

[2] M. Mori, Y. Kosaka, M. Watanabe, H. Nakamura, and M. Kimoto, "A reconciled estimate of the influence of Arctic sea-ice loss on recent Eurasian cooling," Nat Clim Change, vol. 9, pp. 123-129, 2019.

[3] C. S. Bretherton, C. Smith, and J. M. Wallace, "An Intercomparison of Methods for Finding Coupled Patterns in Climate data," J Climate, vol. 5, pp. 541-560, 1992.

[4] J. M. Wallace, C. Smith, and C. S. Bretherton, "Singular value decomposition of wintertime sea surface temperature and 500-mb height anomalies," J Climate, vol. 5, pp. 561-576, 1992.

[5] J. Screen and R. Blackport, "Is sea-ice-driven eurasian cooling too weak in models?," Nature Climate Change, vol. 9, pp. 934-936, 2019. 


\title{
Eurasian cooling in response to Arctic sea ice loss is not proved by maximum covariance analysis (Supplementary Information)
}

\author{
Giuseppe Zappa ${ }^{1,2}$, Paulo Ceppi ${ }^{3}$, and Theodore G. Shepherd ${ }^{1}$ \\ ${ }^{1}$ Department of Meteorology, University of Reading, Reading RG6 6BB, UK \\ ${ }^{2}$ Istituto di Scienze dell'Atmosfera e del Clima, Consiglio Nazionale delle Ricerche \\ (ISAC-CNR), Bologna 40129, Italy \\ ${ }^{3}$ Grantham Institute for Climate Change and the Environment, Imperial College, London \\ $S W 72 A Z, U K$
}

\section{MCA: a brief overview}

Maximum covariance analysis (MCA) enables to identify pairs of spatial patterns that covary in time between two fields $(A$ and $B)$ and that explain as much as possible of the covariance between the two. $A$ and $B$ can have different spatial dimensions $(x)$, but are restricted to having the same temporal dimension $(t)$. Without loss of generality, the fields $A$ and $B$ can be described as two bi-dimensional matrices having dimensions, respectively, $X_{a} \times T$ and $X_{b} \times T$. A set of pairs of co-varying spatial patterns is then identified by decomposing the cross-covariance matrix $(\mathrm{C})$ between $A$ and $B$ via its singular value decomposition:

$$
C=\frac{1}{T} A B^{T}=U \Sigma V^{T}=\sum_{k} \sigma_{k} \cdot \boldsymbol{u}_{\boldsymbol{k}} \boldsymbol{v}_{\boldsymbol{k}}^{T}
$$

where $U=\left(\boldsymbol{u}_{1}, \ldots, \boldsymbol{u}_{N}\right)$ and $V=\left(\boldsymbol{v}_{1}, \ldots, \boldsymbol{v}_{N}\right)$ are squared matrices containing the so-called left $\left(\boldsymbol{u}_{\boldsymbol{k}}\right)$ and right singular vectors $\left(\boldsymbol{v}_{\boldsymbol{k}}\right)$ in their columns. For any value of $k$, each pair of left and right singular vectors describe a mode of co-variability between $A$ and $B . \Sigma$ is a diagonal rectangular matrix, with the values on the diagonal $\left(\sigma_{k}\right)$ reflecting the magnitude of the squared covariance between $A$ and $B$ explained by each mode. Each successive mode following $k=1$ explains a smaller fraction of squared covariance.

The time variability associated with a co-varying mode can be described in terms of expansion coefficients (ECs) $\left(a_{k}\right.$ and $\left.b_{k}\right)$ obtained by projecting the original fields on their own singular vectors, i.e. $a_{k}=\boldsymbol{u}_{\boldsymbol{k}}^{T} A$ and $b_{k}=\boldsymbol{v}_{\boldsymbol{k}}^{T} B$. Since the ECs are computed via a projection, the expansion coefficient $a_{k}$ also incorporates variance from any internal mode of variability in $A$ that is not orthogonal to the singular 
vector $u_{k}$. In the same way, the expansion coefficient $b_{k}$ includes variance due to any mode of internal variability in $B$ not orthogonal to $v_{k}$. The presence of such internal variance in $a_{k}$ and $b_{k}$ has implications for the interpretation of the spatial patterns associated with the expansion coefficients via linear regression. In particular, the patterns obtained by regressing each original field onto their own expansion coefficients (called homogeneous maps) tend to reflect both the structure of the co-varying mode and of any internal modes that contribute to the variance of $a_{k}$ and $b_{k}$. As a result, homogeneous regression maps do not necessarily have a direct relationship to the singular vectors, which exclusively contain the structure of the co-varying mode. After appropriate scaling (see below), the structure of the co-varying mode can instead be recovered via heterogeneous regression maps, so-called because they are obtained by regressing each field on the expansion coefficient of the other field [1].

\section{Scaling of singular vectors and regression maps}

In Fig 1 of the main text, the homogeneous and heterogeneous regression maps, as well as the singular vectors, are scaled to correspond to a one standard deviation anomaly in the ECs. In particular, the scaled $\left(^{\sim}\right)$ expansion coefficients and singular vectors are computed as:

$$
\begin{aligned}
\tilde{a}_{k} & =a_{k} / \sigma_{a_{k}} \\
\tilde{b}_{k} & =b_{k} / \sigma_{b_{k}} \\
\tilde{\boldsymbol{u}}_{k} & =\boldsymbol{u}_{\boldsymbol{k}} \cdot \sigma_{a_{k}} \\
\tilde{\boldsymbol{v}}_{k} & =\boldsymbol{v}_{\boldsymbol{k}} \cdot \sigma_{b_{k}},
\end{aligned}
$$

where $\sigma_{a_{k}}$ and $\sigma_{b_{k}}$ are the standard deviations of the respective ECs. For each grid point, the homogeneous regression maps are obtained as the slope $(m)$ of the linear regressions passing through zero, between each field and their own scaled expansion coefficients. Using the notation $m=<y, x>$ to indicate the slope of y regressed on $\mathrm{x}$ and the standard formulas for least square regression:

$$
\begin{aligned}
& <A, \tilde{a}_{k}>=\frac{A \tilde{a}_{k}^{T}}{\tilde{a}_{k} \tilde{a}_{k}^{T}}, \quad \text { homogeneous map for } \mathrm{A} \\
& <B, \tilde{b}_{k}>=\frac{B \tilde{b}_{k}^{T}}{\tilde{b}_{k} \tilde{b}_{k}^{T}}, \quad \text { homogeneous map for } \mathrm{B}
\end{aligned}
$$

The heterogeneous regression maps can be interpreted in a similar way, but the linear regressions are computed conditional on the EC from the other field:

$$
\begin{aligned}
& <A, E\left(\tilde{a}_{k} \mid \tilde{b}_{k}\right)>=\frac{A \tilde{b}_{k}^{T}}{\tilde{b}_{k} \tilde{b}_{k}^{T}} \cdot \frac{1}{r\left(a_{k}, b_{k}\right)}, \quad \text { heterogeneous map for A } \\
& <B, E\left(\tilde{b}_{k} \mid \tilde{a}_{k}\right)>=\frac{B \tilde{a}_{k}^{T}}{\tilde{a}_{k} \tilde{a}_{k}^{T}} \cdot \frac{1}{r\left(a_{k}, b_{k}\right)}, \quad \text { heterogeneous map for B }
\end{aligned}
$$

$r\left(a_{k}, b_{k}\right)$ is the correlation between the two expansion coefficients, and it represents the scaling factor needed to make the scaled heterogeneous regressions equivalent to the scaled singular vectors. 


\section{A simple model of Arctic--midlatitude interac- tions}

The ability of the approach from M19 to identify climate impacts from sea-ice variability is tested on the output from a simple statistical model of Arctic and Eurasian temperature variability, in which the role played by sea ice can be directly controlled. Despite its simplicity, the statistical model, which is defined as follows, incorporates the key role of sea ice and circulation to force the temperature anomalies discussed in M19:

$$
\begin{aligned}
& \left.\begin{array}{lll}
T_{\text {Arctic }}=-\alpha I & +\beta C+\epsilon_{\text {Arctic }} \\
T_{\text {Asia }}=0 & -\beta C+\epsilon_{\text {Asia }}
\end{array}\right\} \quad \text { Real world } \\
& \hat{T}_{\text {Arctic }}=-\hat{\alpha} I+\hat{\beta} \hat{C}+\hat{\epsilon}_{\text {Arctic }} \\
& \hat{T}_{\text {Asia }}=0 \quad-\hat{\beta} \hat{C}+\hat{\epsilon}_{\text {Asia }}
\end{aligned}
$$

$T_{\text {Arctic }}$ and $T_{\text {Asia }}$ represent, respectively, the surface temperature anomalies in the Arctic and Central Eurasia, I represents the sea-ice anomaly in the Barents and Kara region and $C$ represents the atmospheric circulation anomaly associated with the WACE mode, so that a positive value in $C$ corresponds to an anticyclonic circulation anomaly in Siberia. $\alpha$ and $\beta$ are parameters quantifying the respective influence of sea ice and circulation anomalies on temperature anomalies. For simplicity, it is here assumed that circulation anomalies drive temperature anomalies of equal amplitude, but opposite sign, between the Arctic and Eurasia. $\epsilon$ represents additional variability in the surface temperature, here modelled as Gaussian white noise, that is induced by other local processes or by other atmospheric processes than the WACE mode. The variables representing the output from AMIP simulations are denoted with a . The distinction between the real-world and AMIP-world equations comes from the asymmetry in the system introduced by $I$, as it is the real-world sea ice that affects Arctic surface temperature in both the real world and the AMIP world.

Different possible setups are considered based on the interaction between sea ice and circulation anomalies. First, to reflect the conclusions from M19, we consider a setup in which sea ice drives the probability of occurrence of the WACE mode. In this setup, we define $I$ as an independent random variable, and circulation (both $C$ and $\hat{C}$ ) as a random variable whose mean expected value depends on sea ice:

$$
\left.\begin{array}{l}
I \quad \stackrel{i i d}{\sim} N(0,1) \\
C \mid I \sim N(-\gamma \cdot I, 1) \\
\hat{C} \mid I \sim N(-\hat{\gamma} \cdot I, 1),
\end{array}\right\} \quad \text { Sea ice drives circulation }
$$

where $N(0,1)$ is a normal distribution of zero mean and unit variance, and $\gamma$ and $\hat{\gamma}$ are positive parameters describing the strength of the interaction in the real world and in the AMIP world, respectively. The minus sign before $\gamma$ reflects the conclusion from M19 that a negative anomaly in the sea ice can force a positive WACE mode. This simple model does not include the possibility that circulation or surface temperature are forced by the variability in the SSTs. This is acceptable for the purpose of this 
note since M19 suggest that SSTs play only a minor role in the forcing of the WACE mode.

As a contrasting case, we consider an opposite setup in which it is circulation that drives sea-ice variability, rather than vice-versa. In this case, $C$ - as well as $\hat{C}$ - is modelled as an independent random process, and $I$ as a random variable whose mean value depends on $C$ in the real world:

$$
\left.\begin{array}{ll}
C & \stackrel{i i d}{\sim} N(0,1) \\
\hat{C} & \stackrel{i i d}{\sim} N(0,1) \\
I \mid C & \sim N(-\gamma \cdot C, 1) .
\end{array}\right\} \quad \text { Circulation drives sea ice }
$$

As in the previous setup, $\gamma$ is defined to be positive and it describes the strength of the interaction. The minus sign before $\gamma$ implies that a positive anomaly in C, i.e. a Siberian anticyclone, drives a reduction in sea ice, for example by advecting warm air into the Arctic. The two setups described above become identical for $\gamma=0$, which describes a third setup in which there is no interaction between the variability in the circulation and in the sea ice.

For any given parameter setting, the statistical model is used to generate two sets of synthetic time series of $10^{6}$ values in the variables $T_{\text {Arctic }}, \hat{T}_{\text {Arctic }}, T_{\text {Asia }}, \hat{T}_{\text {Asia }}, C$, $\hat{C}$ and $I$. The same MCA adopted in M19 is applied to the two 2-dimensional time series $\left(T_{\text {Arctic }}, T_{\text {Asia }}\right)$ and $\left(\hat{T}_{\text {Arctic }}, \hat{T}_{\text {Asia }}\right)$ in order to test whether the approach is able to discriminate between cases with different values of $\gamma$ and with different directions of interaction between circulation and sea ice. In particular, three setups of the model's parameters are considered and discussed in Table 1 of the main paper: one in which sea ice drives circulation (with $\gamma=1$ ), one in which there is no interaction between the sea ice and the circulation anomalies $(\gamma=0)$, and one in which it is the atmosphere that drives the sea ice (also with $\gamma=1$ ). For simplicity, a no-bias configuration has been chosen for the other parameters in the statistical model, i.e. $\hat{\gamma}=\gamma, \hat{\alpha}=\alpha=1$ and $\hat{\beta}=\beta=1$.

Finally, we note that simple models such as this one could be extended by future research to account for two-way interaction between sea ice and circulation. Such a tool can be a useful testbed to explore the ability of statistical methods to extract the impact of the lack of atmosphere-ocean coupling in the AMIP simulations.

\section{Significance testing}

The statistical significance of the homogeneous and heterogeneous regression maps is evaluated via boostrapping the individual years with replacement (1000 samples). In particular, we randomly resample with replacement the predictand, i.e. variables $A$ and $B$ in eq. 3 and eq. 4 , while keeping the expansion coefficients unaltered. In each bootstrap sample, the same set of random years is selected across all the AMIP ensemble members. At any given grid point, the regression map is deemed significant at the $5 \%$ level if the observed regression value lies outside the 2.5-97.5 percentile range 
of the bootstrap distribution. Significance is illustrated as stippling in the regression maps.

The statistical significance of the mode of co-variability itself is evaluated by bootstrapping the SVD. As above, the bootstrapping is based on resampling with replacement the years from the AMIP simulations, while keeping the ERA-Interim dataset unaltered. In particular, three metrics of co-variability are considered [2]:

- The square of the Frobenius norm of the cross-covariance matrix $\left(C C=1.9910^{6}\right.$ in the unshuffled dataset).

- The correlation between the ECs ( $R=0.49$ for the first mode).

- The squared covariance fraction $(S C F=54 \%$ for the first mode), i.e. the fraction of cross-covariability that is attributed to a specific mode.

It is found that both $C C$ and $R$ are significantly different from zero at the $1 \%$ level (they lie outside the 0.5-99.5 percentiles of the bootstrap distribution). This strongly suggests that there is significant co-variability between the ERA-Interim and AMIP Eurasian surface temperature fields (significant $C C$ ) and that the first mode detected by the maximum covariance analysis is also statistically significant (significant $R$ ). The same is not true for the $S C F$, which is found to be not statistically significant (p-value $>0.1$. As discussed in reference [2], this does not undermine the significance of the identified mode, since an insignificant $S C F$, with a significant $C C$ and $R$, is to be expected when the variability in each of the two fields is dominated by the leading EOFs.

\section{Sensitivity of the MCA to averaging the ensem- ble members}

In the standard setup from M19, which is also adopted in this comment, the 12 initial-condition ensemble members available for each climate model are concatenated to increase the sample size in the dataset. In Fig. 1, we test the sensitivity of the results to averaging, rather than concatenating, the different initial-condition ensemble members. This setup reduces the unforced internal variability in the dataset, thus enabling to test, via comparison against the standard setup, the impact that internal variability exerts on the output of the maximum covariance analysis. The results show that the AMIP homogeneous map becomes more similar to the heterogeneous one, and that the temperature and circulation signatures of the WACE mode become less evident. This strongly supports that the WACE mode identified in the homogeneous map of M19 reflects the aliasing of internal variability rather than a forced response to Arctic sea ice, as it is discussed in this comment. 
a) ERAI - homogeneous map

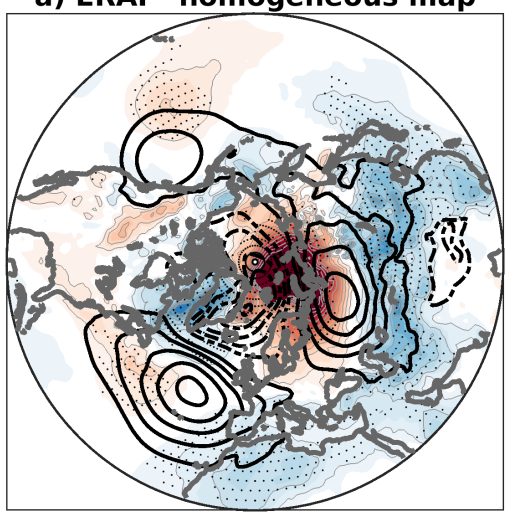

c) ERAI - heterogeneous map

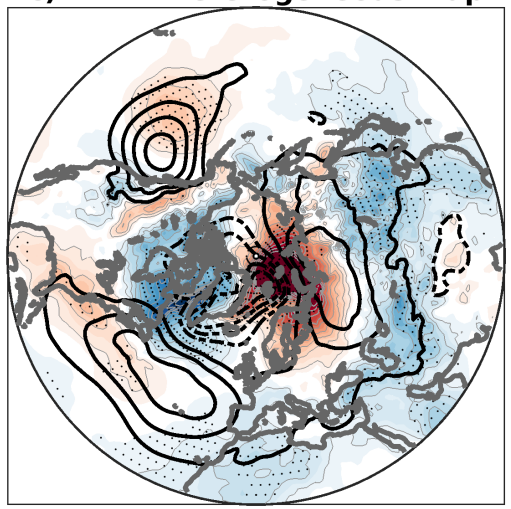

e) ERAI - singular vector

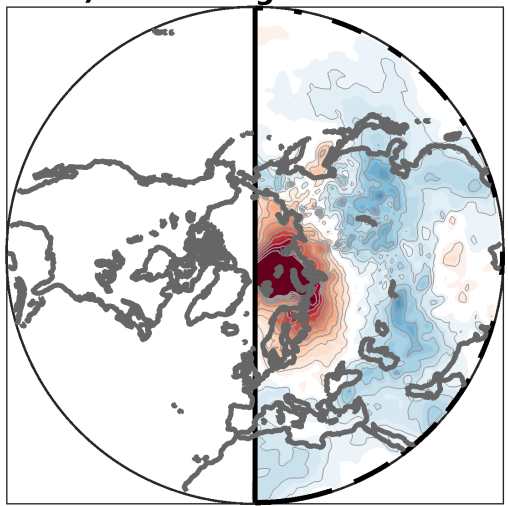

b) AMIP - homogeneous map

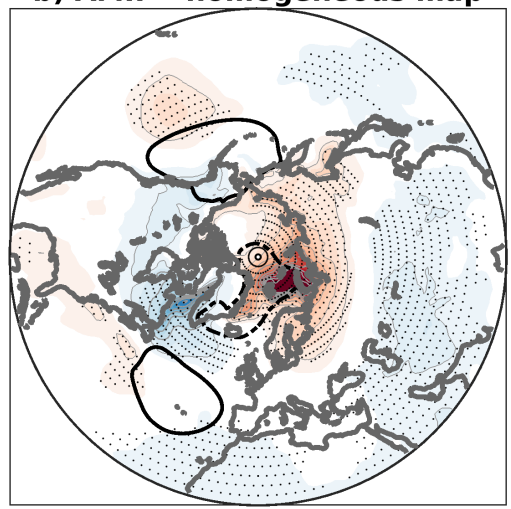

d) AMIP - heterogeneous map

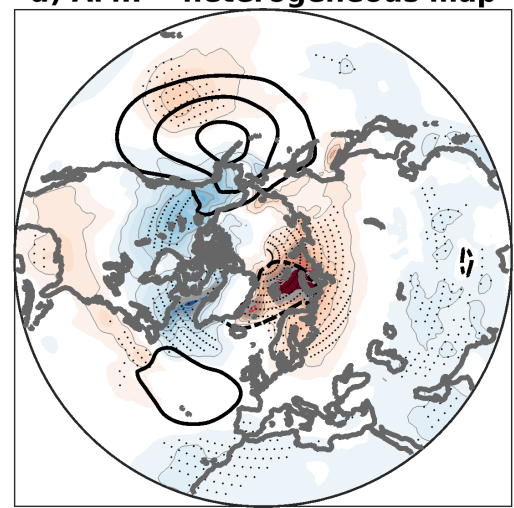

f) AMIP - singular vector

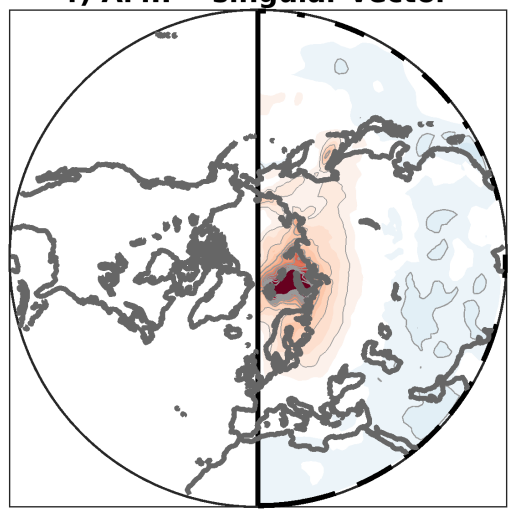

$\begin{array}{lllllllllllllllllllll}-2 & -1.8 & -1.6 & -1.4 & -1.2 & -1 & -0.8 & -0.6 & -0.4 & -0.2 & 0 & 0.2 & 0.4 & 0.6 & 0.8 & 1 & 1.2 & 1.4 & 1.6 & 1.8 & 2\end{array}$

Supplementary Figure 1: Sensitivity of the results to reducing the internal variability in the model data. Panels a-d) are as in Fig. 1a-d of the main paper, but the initial-condition ensemble members are averaged, rather than concatenated, before performing the maximum covariance analysis. Panels e-f) show the corresponding singular vectors. Note how the signals in the AMIP homogeneous map become weaker compared to Fig. 1b of the main paper, which reflects the aliasing of internal variability in the standard setup by M19. 


\section{Confounding influences from SST variability}

The heterogeneous maps shown in Fig 1c-f of the main paper reveal an hemispheric pattern of circulation and temperature anomalies associated with the co-varying mode including, e.g., a weakening of the Aleutian low and a cold anomaly in northern North America. These anomalies may not be statistically significant but they still stand out as some of the largest signals covarying with the first mode from the maximum covariance analysis across the Northern Hemisphere. At the same time, the co-varying mode is also associated with sea-surface temperature anomalies including, e.g., a warm anomaly in the North Pacific and an ENSO-like pattern in the tropical Pacific (Extended Data Fig. 3). Here, we suggest that these SST anomalies can potentially force a sizeable portion of the co-varying circulation signals without the need to invoke an influence from Arctic sea ice. As a case study, Fig. 2 compares the surface temperature, sea-level pressure and geopotential height at $500 \mathrm{hPa}$ associated with the co-varying mode against those associated with ENSO. The latter is obtained by regressing the output from the AMIP simulations on the Nino 3.4 index (area-averaged surface temperature between $5 \mathrm{~S}-5 \mathrm{~N}$ and $170 \mathrm{~W}-120 \mathrm{~W}$ ). Despite a correlation of only 0.2 between the ECs and the Nino 3.4 index, there is good resemblance between the ENSO-driven circulation patterns and those associated with the co-varying mode over the North Pacific and North American sectors. This illustrates how even a small and possibly just accidental - correlation with an internal mode of variability can act as a substantial confounding factor in the attempt to isolate the role of Arctic sea-ice variability in the climate system. Fig. 2a also suggests that these ENSO-like SST anomalies may modulate the southern Eurasian surface temperature variability too, though the signal appears to be weak. Quantifying their exact role, as well as that of the SSTs from the other ocean basins, will require the application of a dedicated experimental framework which should be a topic for future research. 
a) ts AMIP regressed on Nino 3.4

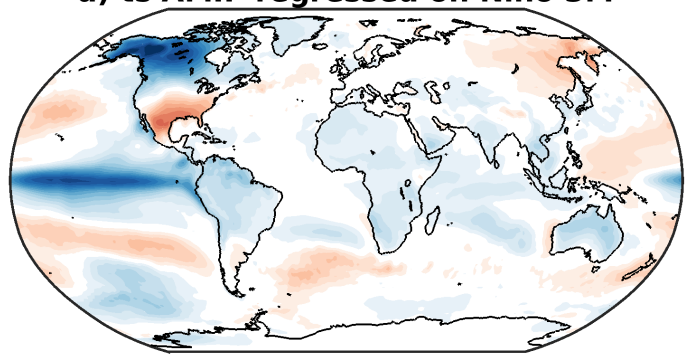

c) psI AMIP regressed on Nino 3.4

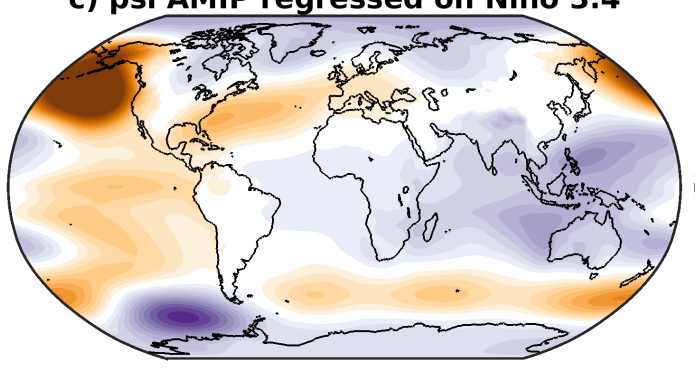

e) z500 AMIP regressed on Nino 3.4

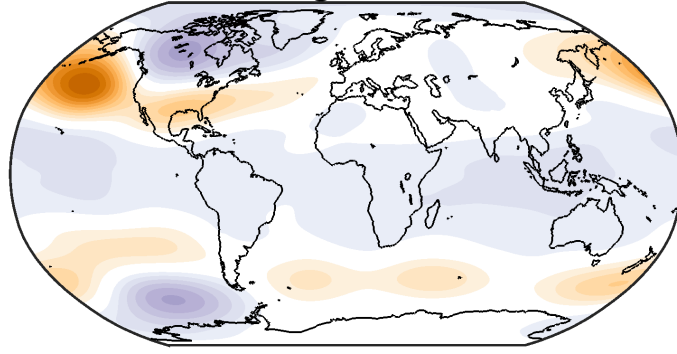

b) ts AMIP heterogeneous map
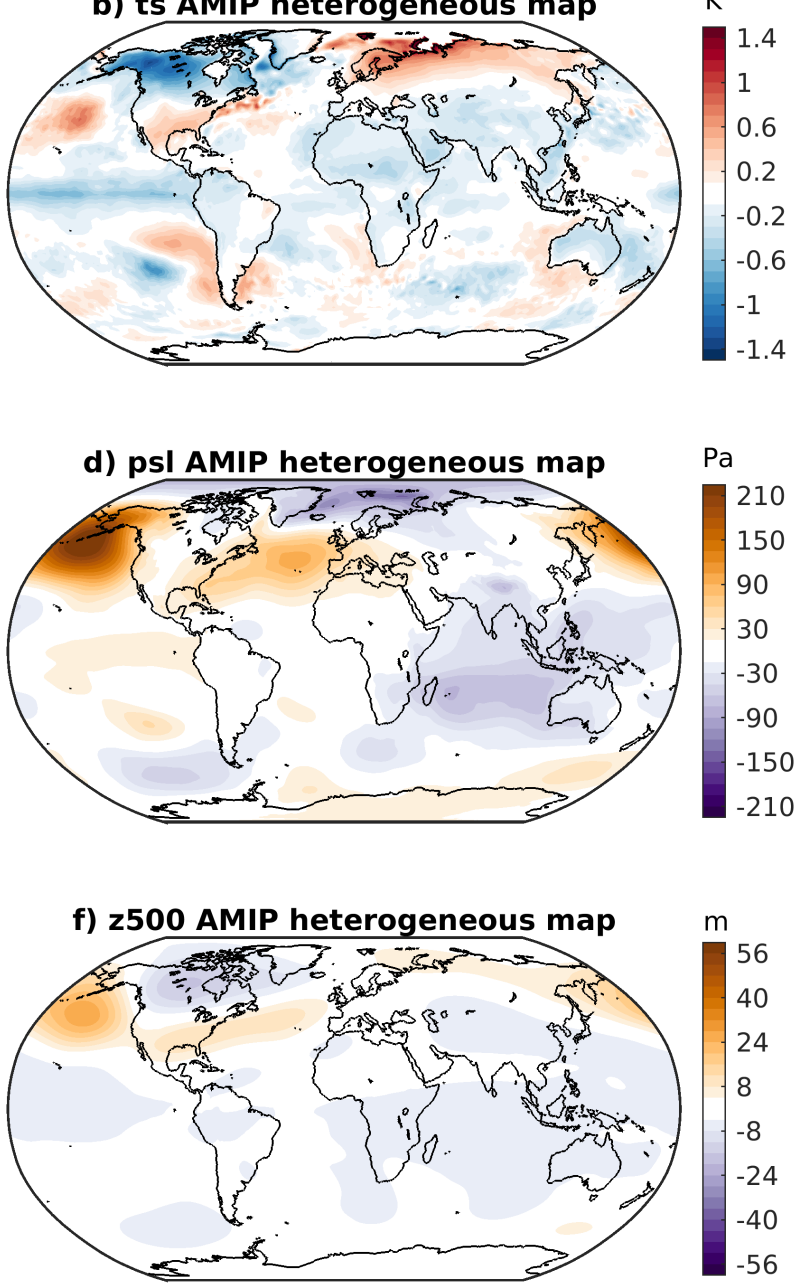

Supplementary Figure 2: Confounding influences from SST variability. Comparison of a) the regression of winter-mean (DJF) surface temperature on the Nino 3.4 index in the AMIP simulations (sign reversed), against the b) heterogeneous map of the co-varying mode in the AMIP simulations. Surface temperature is defined as the sea-surface temperature over the ocean, and as 2-meter air temperature over land. c-d) same as a-b) but for sea level pressure and e-f) same but for geopotential height at $500 \mathrm{hPa}$. All regressions are scaled to correspond to one-sigma anomaly in the predictor. Panels e) and f) are based on 5 AMIP models since upper-air data is unavailable for GFDL-AM3. 


\section{References}

[1] C. S. Bretherton, C. Smith, and J. M. Wallace, "An Intercomparison of Methods for Finding Coupled Patterns in Climate data," J Climate, vol. 5, pp. 541-560, 1992.

[2] J. M. Wallace, C. Smith, and C. S. Bretherton, "Singular value decomposition of wintertime sea surface temperature and 500-mb height anomalies," J Climate, vol. 5, pp. 561-576, 1992. 